\title{
Noise and regeneration in semiconductor waveguides with saturable gain and absorption
}

\author{
Öhman, Filip; Bischoff, S.; Tromborg, Bjarne; Mørk, Jesper
}

Published in:

I E E E Journal of Quantum Electronics

Link to article, DOI:

10.1109/JQE.2003.823035

Publication date:

2004

Document Version

Publisher's PDF, also known as Version of record

Link back to DTU Orbit

\section{Citation (APA):}

Öhman, F., Bischoff, S., Tromborg, B., \& Mørk, J. (2004). Noise and regeneration in semiconductor waveguides with saturable gain and absorption. I E E E Journal of Quantum Electronics, 40(3), 245-255.

https://doi.org/10.1109/JQE.2003.823035

\section{General rights}

Copyright and moral rights for the publications made accessible in the public portal are retained by the authors and/or other copyright owners and it is a condition of accessing publications that users recognise and abide by the legal requirements associated with these rights.

- Users may download and print one copy of any publication from the public portal for the purpose of private study or research.

- You may not further distribute the material or use it for any profit-making activity or commercial gain

- You may freely distribute the URL identifying the publication in the public portal 


\title{
Noise and Regeneration in Semiconductor Waveguides With Saturable Gain and Absorption
}

\author{
Filip Öhman, Svend Bischoff, Bjarne Tromborg, and Jesper Mørk
}

\begin{abstract}
We have modeled the noise properties of a novel waveguide device with regenerative properties. The device consists of alternating sections of saturable gain and absorption, which give a nonlinear power transfer function. We investigate the relative intensity noise spectra and signal-to-noise ratio after the device by both a small-signal analysis and large-signal simulation, and we show that the gain saturation gives noise redistribution at the mark level. We also examine the influence of the nonlinearity on the noise probability density function and show that the standard approximations of Gaussian and noncentral $\chi^{2}$ distributions do not give a satisfying description. The strength and weaknesses of the limiting cases of static transfer functions and linear noise transfer as well as the small-signal analysis are examined in the case of bit-error-rate estimation in a cascade of regenerators. The interplay between increased nonlinearity and noise is investigated and we show that the increased nonlinearity achieved by additional device sections can improve the cascadability although more amplified spontaneous emission noise is added.
\end{abstract}

Index Terms-All-optical signal processing, noise, optical communication, regeneration, semiconductor optical amplifiers (SOAs).

\section{INTRODUCTION}

A LL-OPTICAL networks require a number of signal processing functionalities, such as regeneration, wavelength conversion, and switching [1]. Regeneration is necessary since the optical signal is degraded in the system due to, for example, losses, dispersion, and noise. It is common to divide regeneration into three functionalities. Reamplification is the use of optical amplifiers in order to compensate for fiber attenuation and coupling losses. Reshaping implies the improvement of the optical signal-to-noise ratio (OSNR) by, for example, extinction ratio improvement, noise redistribution, and, in the case of pulsed systems, pulse compression. These improvements are usually achieved by a component with a nonlinear intensity transfer function, where the limit of a step function or complete regeneration is equivalent to making a decision between a logical one and zero. Finally, retiming is used for reducing timing jitter and can be accomplished by transferring the data signal to a new low-jitter pulse train. The

Manuscript received June 13, 2003; revised November 14, 2003. This work was supported by the IST Project BIGBAND and the Danish Technical Research Council through the SCOOP Program.

F. Öhman, B. Tromborg, and J. Mørk are with the Research Center for Communications, Optics and Materials (COM), Technical University of Denmark, DK-2800 Kgs. Lyngby, Denmark (e-mail: fo@com.dtu.dk).

S. Bischoff is with Alight Technologies A/S, DK-3520 Farum, Denmark.

Digital Object Identifier 10.1109/JQE.2003.823035 three functionalities (reamplification, reshaping, and retiming) together are usually referred to as 3R-regeneration, while in 2R-regeneration the retiming is omitted.

Semiconductor optical amplifier (SOA)-based devices offer many optical signal processing functionalities. A promising principle of operation employs cross phase modulation in SOA-based interferometers [2]-[5]. In the case of regeneration, this can be accomplished both with and without wavelength conversion. In the case of wavelength conversion, the input signal modulates light at a new wavelength provided by the regenerator. This allows for retiming (3R) of the signal if clock recovery and an optical clock are provided. In a pass-through setup, i.e., without wavelength conversion, the input signal is regenerated (2R) by self-modulation. One example of wavelength conversion and regeneration in SOA-based devices can be found in [3] while pass-through regenerators are demonstrated in [2], [4], and [5].

In this study, we will examine a simple pass-through device with regenerative properties (2R). It consists of a combination of SOAs and electroabsorbers (EAs). The basic idea of the device is to use the gain saturation in the SOA combined with the absorption bleaching in the EA to achieve a simple and potentially cheap device with regenerative properties. This type of device, with a single amplifier-absorber pair, has been proposed earlier for regeneration [6], but also for chirp control in modulators [7] and compensation of waveform distortion in SOAs [8]. The addition of the EA to the SOA can thus potentially remedy some of the shortcomings of the SOA by sacrificing some gain of the device. One may argue that, since amplifiers will be needed anyway, one may as well improve the performance by using amplifier-absorber combinations. We will extend the investigation to several SOA-EA pairs in order to get a stronger nonlinearity and focus on the redistribution of intensity noise in the devices.

The objective of the investigation is to analyze and compare different methods to evaluate the regenerative properties of the single SOA-EA device and of cascaded devices. The true test of a regenerator is the bit-error-rate (BER) evolution in a cascade of fiber links and regenerators. We study four methods to calculate this evolution: a small-signal noise analysis, a Monte-Carlo type of large-signal noise simulation, a static transfer function approach, which is a useful approximation in the low bit-rate limit, and a linear transfer function approach, which is aimed for the high-bit-rate limit.

The noise of the output signal arises from the transformed noise of the input signal as well as from amplified spontaneous emission (ASE) in the SOA. Noise in SOAs has been investigated extensively, experimentally [9], as well as theoretically. 
The theoretical work ranges from small-signal analyses of linear gain models [10] to models including gain saturation [11]. We use a small-signal analysis of the SOA and EA Langevin rate equations to derive the noise spectra of the output signal, and we show that the spectra are in very good agreement with the spectra obtained from a large-signal simulation of the equations. However, the Gaussian or noncentral $\chi^{2}$ probability density functions (pdfs) for the noise distributions of the mark and space derived from the small-signal analysis are not in good agreement with the pdfs from the large-signal simulation. The two methods will for these nonlinear devices lead to different predictions for the BER. This is in contrast to previous examples showing a remarkable agreement between the BER obtained from Gaussian pdfs and the BER obtained from more precise pdf calculations [12], [13]. The large-signal simulation will produce pdf curves and BERs consistent with the device models, but the practical limitations in computing time prevents test down to BERs of interest for telecom applications $\left(\mathrm{BER} \approx 10^{-9}\right)$.

Assuming a transfer function giving the output power $P_{\text {out }}$ in terms of the input power $P_{\text {in }}$ allows a simple calculation of the pdfs of the output signal in terms of the pdf of the input signal. In order to analyze the nonlinear properties but still limit the calculation effort and time, even for a large number of cascaded devices, the devices can be represented by their static transfer functions and the noise redistribution is thus easily calculated for a large number of cascaded devices [14], [15]. By using partially linear transfer properties in an adequate way, the calculations can be simplified and give relevant results, for both wavelength converters [16] and regenerators [17]. We will argue that the linear transfer function $P_{\text {out }}=$ const. $* P_{\text {in }}$ through the mark operation point is valid for high-bandwidth signals. We show that the signal-to-noise ratio (SNR) from the small-signal analysis agrees with the linear transfer function calculation in the limit of large-bandwidth signals and with the static transfer function calculation in the limit of narrow-bandwidth signals.

In Section II, we briefly describe the used models. The noise properties of individual SOAs and EAs are discussed in Section III in order to understand the noise added and the dynamics of the noise redistribution. We then continue with a short description of our proposed SOA-EA device and its noise properties in Section IV. Both the single devices and the SOA-EA device is compared to the limiting cases of linear and static transformation. In order to get closer to our goal of BER estimations, investigations of the influence of nonlinear noise redistribution on the probability density functions of the noise is performed in Section V. The cascadability of the regenerators is then finally examined using different methods in Section VI. Finally, Section VII summarizes our results and draws the main conclusions.

\section{MODELS}

This section describes the most relevant parts of the different models we use. The device model used in our calculations is a standard rate-equation model for the carrier density in an SOA and a propagation equation for the electric field, described in [18]. The model takes gain and absorption saturation into account. The analysis of noise in the SOA is performed in two different ways, a small-signal analysis and a statistical simu- lation. In both cases, the noise model is based on the same basic assumptions. The noise is thus incorporated by Langevin forces, with correlation functions according to [11] and [19] to the equations. The resulting equations for carrier density and optical field are

$$
\begin{aligned}
& \frac{\partial N}{\partial t}=\frac{I}{q V}-\frac{N}{\tau_{s}}-\frac{g(N)}{A \hbar \omega_{0}}|\tilde{E}|^{2}+\tilde{f_{N}} \\
& \frac{\partial \tilde{E}}{\partial z}=\frac{1}{2}\left(g(1-i \alpha)-\alpha_{\text {int }}\right) \tilde{E}+\tilde{f_{E}}
\end{aligned}
$$

where $I$ is the injected current, $q$ is the elementary charge, $V$ is the active volume, $\tau_{s}$ is the spontaneous carrier lifetime (or sweep-out time for the EA), $A$ is the effective cross-sectional area of the active region, $\hbar \omega_{0}$ is the photon energy, $\alpha$ is the linewidth enhancement factor, $\alpha_{\text {int }}$ is the waveguide losses, and $\tilde{E}(t, z)$ is the complex envelope of the optical field normalized such that $|\tilde{E}|^{2}$ is the power. The time variable $t$ is a shifted time coordinate, $t=t_{\text {real }}-z / v_{g}$, where $t_{\text {real }}$ is the real time coordinate and $v_{g}$ is the group velocity. The gain $g(N)$ is approximated as a linear function of the carrier density and it is assumed that the carrier frequency $\omega_{0}$ is chosen at the gain peak. The gain is then

$$
g(N)=a\left(N-N_{0}\right)
$$

where $a$ is the differential modal gain and $N_{0}$ is the carrier density at transperancy. $f_{N}$ and $f_{E}$ are the noise terms (Langevin forces) for the carriers and spontaneous emission, respectively.

The EA is modeled using the same equations as for the SOA. In this case, the characteristic time constant $\tau_{s}$ is an effective carrier sweep-out time describing the time it takes for the photogenerated carriers to be swept out of the active region by the applied electrical field. The absorption is described by a negative value of the gain $(g)$. Both noise terms are assumed to be zero since there is no ASE in the EA and the carrier noise is assumed to be small compared to the ASE in the SOA for the SOA-EA combinations. A more detailed model for the EA dynamics could be used [20]. However, the present model incorporates the main feature of EA saturable absorption and suffices for the present analysis of situations where ultrafast processes like spectral hole burning and carrier heating [21] can be neglected.

\section{A. Small-Signal Analysis}

The first of our two noise descriptions assumes that the noise is small compared to the signal field and is hereafter referred to as the small-signal analysis. This small-signal analysis for calculating the noise spectrum of an SOA was presented by Shtaif et al. [11], to which we refer for further details and derivations. In this study, we will only present, and use, the most relevant results.

The electric field and the noise terms are from now on normalized with the square root of the saturation power $E=\tilde{E} / \sqrt{P_{\text {sat }}}, f_{i}=\tilde{f}_{i} / \sqrt{P_{\text {sat }}}(i=E, N)$, where $P_{\text {sat }}=A \hbar \omega_{0} / a \tau_{s}$. The field is divided into a signal and a noise part according to

$$
\begin{aligned}
E & =E_{s}\left(1+\frac{\delta E}{E_{s}}\right)=\left(\rho_{s}+\delta \rho\right) \exp \left[i\left(\phi_{s}+\delta \phi\right)\right] \\
& \simeq E_{s}\left(1+\frac{\delta \rho}{\rho_{s}}+i \delta \phi\right)
\end{aligned}
$$


where $E_{s}$ is the field without noise, $\rho_{s}$ is its amplitude, and $\phi_{s}$ its phase. $\delta E, \delta \rho$, and $\delta \phi$ are the small deviations from the respective values due to noise. The gain coefficient is, in the same manner, written as

$$
g=g_{s}+\delta g
$$

By substituting these expressions into (1) and (2) and solving for the electric field in the frequency domain, we obtain

$$
\begin{aligned}
\frac{\delta E(L)}{E_{s}(L)}= & \frac{\delta \rho}{\rho_{s}}+i \delta \phi \\
= & \frac{\delta E(0)}{E_{s}(0)}+(1-i \alpha)(H(0)-1) \frac{\delta \rho(0)}{\rho_{s}(0)} \\
& +\int_{0}^{L}\left((1-i \alpha) N_{\rho}(H(z)-1)+N_{E}\right) d z
\end{aligned}
$$

where $N_{E}=N_{\rho}+i N_{\phi}$ and

$$
\begin{aligned}
N_{\rho}(\omega, z)= & \frac{1}{2} \frac{\tau_{s}}{1+\rho_{s}^{2}+i \omega \tau_{s}} f_{g}(\omega, z) \\
& +\frac{1}{2}\left(\frac{f_{E}(\omega, z)}{E_{s}}+\frac{f_{E}^{*}(-\omega, z)}{E_{s}^{*}}\right) \\
N_{\phi}(\omega, z)= & -\frac{\alpha}{2} \frac{\tau_{s}}{1+\rho_{s}^{2}+i \omega \tau_{s}} f_{g}(\omega, z) \\
& +\frac{1}{2 i}\left(\frac{f_{E}(\omega, z)}{E_{s}}-\frac{f_{E}^{*}(-\omega, z)}{E_{s}^{*}}\right)
\end{aligned}
$$

where $f_{i}(\omega, z)$ is the Fourier transform of $f_{i}(t, z)(i=E, g)$ and $f_{g}=a f_{N} . H(z)$ is given as

$$
H(z)=\exp \left(-\int_{z}^{L} \frac{g_{s} \rho_{s}^{2}}{1+\rho_{s}^{2}+i \omega \tau_{s}} d z^{\prime}\right) .
$$

The noise spectra of the SOA can then be calculated using a semiclassical or quantum mechanical treatment of the correlation functions for $f_{g}$ and $f_{E}$. The results show that the different treatments differs only by a shot noise term in the expressions for relative intensity noise (RIN) and phase noise spectra [11]. In this paper, we will only deal with the intensity noise in the form of RIN, which is defined as

$$
\operatorname{RIN}(\omega, z)=\frac{S_{\delta \rho^{2}, \delta \rho^{2}}(\omega, z)}{\rho_{s}^{4}(z)}=4 \frac{S_{\delta \rho, \delta \rho}(\omega, z)}{\rho_{s}^{2}(z)}
$$

where

$$
S_{x, y}(\omega)=\int_{-\infty}^{\infty}\left\langle x^{*}(t) y(t+\tau)\right\rangle e^{i \omega \tau} d \tau
$$

is the cross correlation power spectrum between the two processes $x(t)$ and $y(t)$. The RIN is thus defined as the noise power relative to the mean power squared.

By using these equations and the correlation relations for the Langevin noise terms, the expression for the RIN spectrum can be calculated. The result consists of five terms

$$
\mathrm{RIN}=R_{0}+R_{\mathrm{sp}}+R_{g}+R_{g, \mathrm{sp}}+R_{\mathrm{shot}}
$$

where the different terms represent the noise contribution from the input signal, spontaneous emission, and carriers and the cross correlation between carrier noise and spontaneous emission and shot noise, respectively. The expressions for the noise terms are [11]

$$
\begin{aligned}
R_{0}(\omega)= & |H(0)|^{2}(\operatorname{RIN}(\omega, z=0)) \\
R_{\mathrm{sp}}(\omega)= & \frac{\hbar \omega_{0}}{P_{\mathrm{sat}}} \int_{0}^{L}|H(z)|^{2} \frac{2 g_{s} n_{\mathrm{sp}}}{\rho_{s}^{2}} d z \\
R_{g}(\omega)= & \frac{\hbar \omega_{0}}{P_{\mathrm{sat}}} \int_{0}^{L}|H(z)|^{2} \\
& \cdot \frac{\xi g_{0}+g_{s}+a N_{0}(1+\xi)+g_{s} \rho_{s}^{2}\left(2 n_{\mathrm{sp}}-1\right)}{\left(1+\rho_{s}^{2}\right)^{2}+\left(\omega \tau_{s}\right)^{2}} d z \\
R_{\mathrm{g}, \mathrm{sp}}(\omega)= & -2 \frac{\hbar \omega_{0}}{P_{\mathrm{sat}}} \int_{0}^{L}|H(z)|^{2} \frac{2\left(1+\rho_{s}^{2}\right) g_{s} n_{\mathrm{sp}}}{\left(1+\rho_{s}^{2}\right)^{2}+\left(\omega \tau_{s}\right)^{2}} d z(16) \\
R_{\mathrm{shot}}= & \frac{\hbar \omega_{0}}{P(L)}
\end{aligned}
$$

where $n_{\mathrm{sp}} \approx\left(g+a N_{0}\right) / g$ is the inversion factor and $\xi$ is a parameter that describes the noise of the current source. It is equal to 1 when the current source has shot noise behavior. The small-signal gain $g_{0}$ is defined as $g_{0}=a\left(\tau_{s} I / q V-N_{0}\right)$. The input RIN does not include shot noise. The SNR can now be defined as

$$
\mathrm{SNR}=\left(\int_{-B_{e}}^{B_{e}} \operatorname{RIN}(2 \pi f) d f\right)^{-1}
$$

where $B_{e}$ is the electrical bandwidth of the detector. This definition is identical to the ratio between the mean power squared and the noise variance, in the specified bandwidth.

\section{B. Simulation}

The second noise description assumes a sampled signal in the time domain and includes ASE noise in the SOA by adding a noise field with appropriate statistics. Again, the detailed work has been presented elsewhere [19], and we will only present the equations relevant to our work. The SOA is divided into short sections of length $\Delta z$. The sections are modeled as ideal SOAs by ignoring the noise terms and the internal losses. Equations (1) and (2) can then be rewritten as an ordinary differential equation

$$
\frac{\partial h}{\partial t}=\frac{h_{0}-h}{\tau_{s}}-\frac{P_{\mathrm{in}}(t)}{P_{\mathrm{sat}} \tau_{s}}[\exp (h)-1]
$$

where $h$ is the integrated gain, given as follows:

$$
h(t)=\int_{z}^{z+\Delta z} g(z, t) d z .
$$

$h_{0}=g_{0} \Delta z$ and $P_{\text {in }}(t)$ is the input power. The output field for the ideal section is thus

$$
E_{\text {out }}(t)=E_{\text {in,tot }}(t) \exp \left[\frac{1}{2}(1+i \alpha) h(t)\right] .
$$

The internal losses and noise are modeled by solving the ideal loss-less model for the short sections and then including the losses and noise of the propagating light at the interfaces between the sections. The properties of the noise field are calcu- 
lated from (1) and (2) and the correlation functions of the noise terms according to [19]. The added noise field is

$$
E_{\mathrm{ASE}}=E_{\text {real }}+i E_{\text {imag }}
$$

where $E_{\text {real }}$ and $E_{\text {imag }}$ is the real and imaginary part of the noise field, respectively. They have a Gaussian distribution with zero mean and variance according to

$$
\operatorname{Var}\left[E_{\text {real }}\right]=\operatorname{Var}\left[E_{\text {imag }}\right]=\frac{1}{2} \frac{\hbar \omega_{0} n_{\mathrm{sp}} g_{s} \Delta z}{P_{\text {sat }}} 2 B_{N}
$$

where $B_{N}$ is the equivalent noise bandwidth. $B_{N}$ is introduced in order to make $E_{\mathrm{ASE}}$ a bandlimited white Gaussian noise, since an ideal white noise has infinite bandwidth and hence infinite noise power. In reality, different effects, for example, the gain spectrum of the SOA, limit the ASE bandwidth. Since our model does not include such limiting effects, $B_{N}$ is chosen to be large enough to properly include, i.e., exceed, the modulation bandwidth of the simulated devices. On the other hand, $B_{N}$ has to be limited such that the ASE power does not contribute significantly to the saturation of the SOA, i.e., $B_{N}$ cannot be too large. This limitation is due to the unidirectional nature of the model, i.e., ASE traveling backward in the SOA is not considered. Neglecting this effect will underestimate the noise figure of the SOA, but the effect is small compared to that of saturation from the signal. Accepting this limitation of the model, we choose $B_{N}$ to properly include the bandwidth of the devices and make sure that the ASE power is very small compared to the mean input power. This limitation of the influence of accumulated ASE power when the device is cascaded can be justified by the fact that the absorption in the EA will keep the ASE power low [22]. We also choose to limit the total simulated bandwidth to the same value and use a time-step $\Delta t=1 / 2 B_{N}$ in accordance with the sampling theorem. In order to fulfill these requirements, $B_{N}$ is chosen to be $30 \mathrm{GHz}$ in all the calculations and the values of the input signal SNR ( $\left.\mathrm{SNR}_{\text {in }}\right)$ stated in the paper are defined at this bandwidth.

In practice, $E_{\text {real }}$ and $E_{\text {imag }}$ are constructed by two pseudorandom sequences with zero mean and unit variance that are multiplied by the square root of the right-hand side of (23). The total field including the signal and noise is then used in the model for the ideal sections, i.e.,

$$
E_{\mathrm{in}, \mathrm{tot}}=E_{\mathrm{in}}+E_{\mathrm{ASE}}
$$

where $E_{\text {in }}$ is the input field of the section. The small-signal analysis shows that the carrier noise can be neglected when the intensity noise is considered at reasonable power levels [11], and thus $f_{g}$ is zero in the simulations.

The output field from the model is analyzed statistically to find the SNR and a histogram estimating the pdf. Since the method has the characteristics of a statistical simulation, it is hereafter referred to as the "simulation." For comparison, the static power transfer function of the devices, i.e., the output power from our model given as a function of a $\mathrm{CW}$ input power, is also used to calculate the output signal. The output signal from the static case is then treated in the same way as for the simulated signal. Finally, a linear transfer function, representing an ideal linear amplifier with the same gain as the simulated device, is used for reference. In these two cases, no ASE is added and only the redistribution of the input noise is considered. They are referred to as the "static" and "linear" cases, respectively.

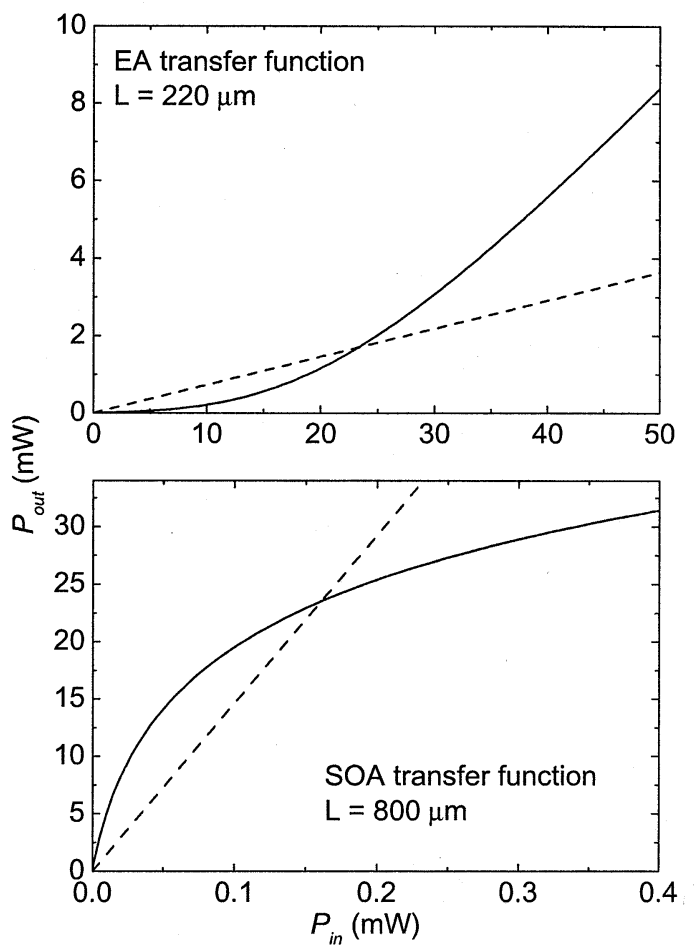

Fig. 1. Transfer function of the modeled SOA and EA (solid line). The nonlinear behavior, due to absorption bleaching and gain saturation, respectively, is clearly seen. The dashed lines show the transfer function of a linear device with the same absorption/gain as the devices at the chosen operating point.

\section{NOISE TRANSFORMATION IN SOAS AND EAS}

In order to simplify the discussion, we first examine a standalone SOA and EA, where we investigate the noise added by the SOA and the dynamics of the noise redistribution in both SOAs and EAs. We also make a comparison between the small-signal analysis and the simulation.

The static power transfer functions, i.e., mean output power plotted versus mean input power, for the EA and SOA are shown in Fig. 1. We have used parameters corresponding to an 800- $\mu \mathrm{m}$-long SOA and $220-\mu \mathrm{m}$ EA. All other parameter values used in the calculations are listed in Table I. Typical parameter values are used, and these may in some cases differ from what is considered state of the art, which will limit the modulation bandwidth of the presented devices. However, all-optical signal processing at $40 \mathrm{GHz}$ and above in both SOAs and EAs has been reported [23], [24]. In the present paper, we emphasize the analysis and understanding of the nonlinear device properties, rather than the optimization of device performance.

From Fig. 1, it is obvious that the transfer functions are not linear, but rather show absorption bleaching in the EA and gain saturation in the SOA. These nonlinearities may be used for reshaping a signal injected into the device and should be considered when the noise properties of the devices are investigated. The noise reduction in a saturated SOA and the extinction ratio improvement and noise reduction by using a saturable absorber have been investigated both experimentally and theoretically [20], [22], [25]-[27].

In order to investigate the added noise and the dynamics of the noise redistribution, we use a mean input power that 
TABLE I

PARAMETER VALUES USED IN THE CALCULATIONS

\begin{tabular}{l|c|c}
\hline \multirow{2}{*}{ Parameter } & \multicolumn{2}{|c}{ Value } \\
\cline { 2 - 3 } & SOA & EA \\
\hline Time constant, $\tau_{\mathrm{s}}[\mathrm{ps}]$ & 100 & 100 \\
Saturation power, $E_{\text {sat }}[\mathrm{pJ}]$ & 2.17 & 0.54 \\
Internal loss, $\alpha_{\text {int }}\left[\mathrm{m}^{-1}\right]$ & 2000 & 2000 \\
Linewidth enhancement factor, $\alpha$ & 5 & 5 \\
Small signal gain, $g_{0}\left[\mathrm{~m}^{-1}\right]$ & 11000 & -19158 \\
Differential gain, $a\left[\mathrm{~m}^{2}\right]$ & $2 \cdot 10^{-20}$ & $8 \cdot 10^{-20}$ \\
Carrier density at transparency, $N_{0}\left[\mathrm{~m}^{-3}\right]$ & $1 \cdot 10^{24}$ & $0.8 \cdot 10^{24}$ \\
\hline
\end{tabular}

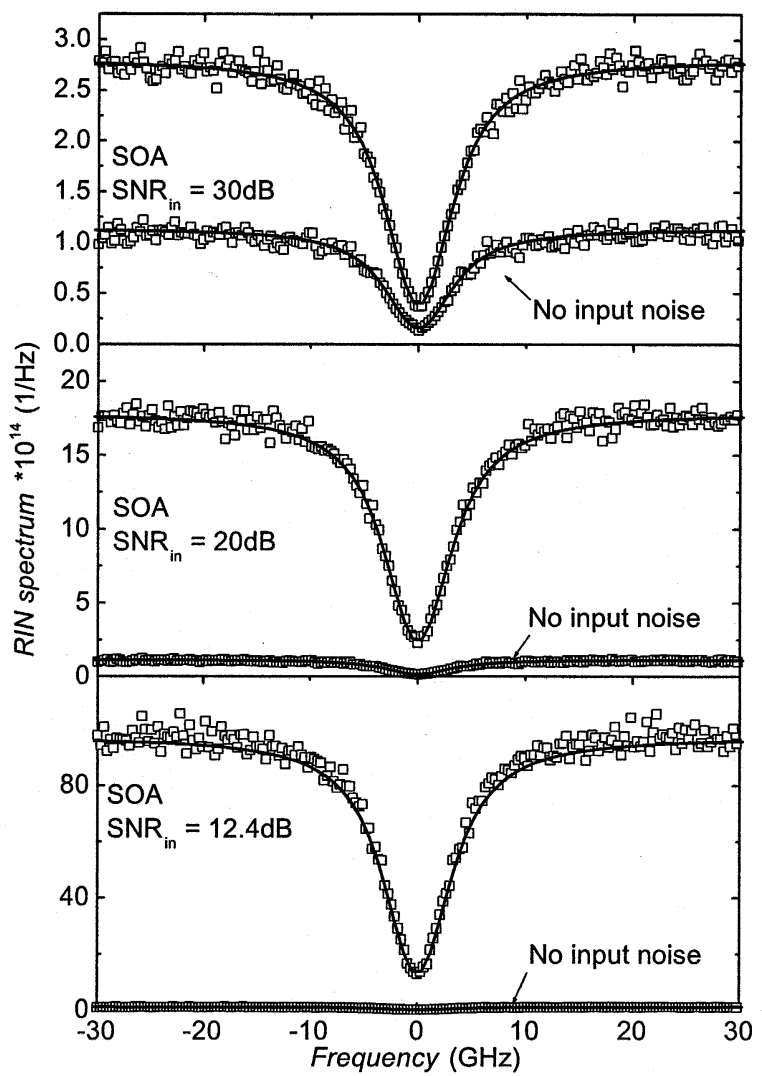

Fig. 2. RIN spectrum after the SOA, showing the noise suppression at low bandwidths due to the nonlinear transfer function. The input power is $0.16 \mathrm{~mW}$ in all cases, representing the "1"-level. The spectrum of the ASE is also shown in order to visualize the relative magnitudes of ASE and input noise at different levels of input noise, $\mathrm{SNR}_{\mathrm{in}}$. The squares are the large-signal simulations and the solid line represents the small-signal analysis.

represents the power levels at the logical " 1 "-level of the combined SOA-EA regenerators discussed later in this article. The input signal is a CW beam with Gaussian bandlimited white noise. Three different values of the SNR of the input signal of $12.4,20$, and $30 \mathrm{~dB}$, at a bandwidth of $30 \mathrm{GHz}$, were chosen to give cases where the noise on the input signal dominates over the ASE of the SOA and conversely. The resulting RIN spectra are shown in Fig. 2 for the SOA and in Fig. 3 for the EA. The solid black line is the result from the small-signal analysis and the squares are the simulated data.

The dip at low frequencies in the RIN spectrum of the SOA and the corresponding bump in the spectrum of the EA are due to the nonlinearities of the transfer functions. In the SOA case, the static transfer function has a slope lower than that of a linear

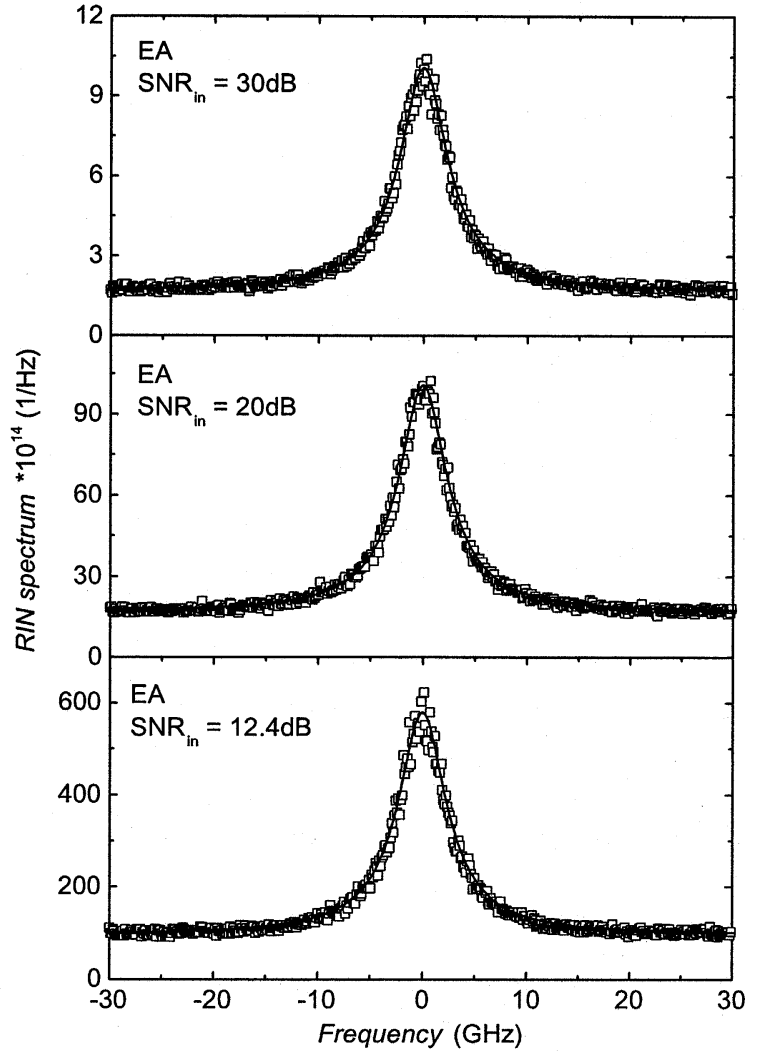

Fig. 3. RIN spectrum after the EA showing the noise enhancement due to the nonlinear transfer function. The input power is $23.4 \mathrm{~mW}$ in all cases, representing the "1"-level. The squares are the large-signal simulations and the solid line represents the small-signal analysis.

device with the same operating point (dashed lines in Fig. 1). This means that the part of the noise distribution above the mean power experiences less gain and the part below somewhat higher gain than the mean. This leads to a redistribution of the noise to a narrower distribution, compared to a linear device. The effect is similar but opposite in the single EA, which leads to a broader noise distribution. The EA does, however, improve the signal quality by noise redistribution at the "0"-level and extinction ratio improvement, as will be discussed in Section IV. Furthermore, it is important to notice that the redistribution is due to gain saturation from carrier density depletion in the SOA and absorption bleaching due to band filling and screening in the EA. It is therefore dependent on the carrier dynamics, and the finite characteristic response time of the devices means that the redistribution only affects the low-frequency components of the noise for both the SOA and the EA, hence the dip and bump in the spectrum. The corresponding RIN spectrum for the linear power transformation with the same gain/absorption would produce a flat spectrum at the level of the high-frequency part of the SOA and EA. If, on the other hand, the static transfer functions were used, i.e., the noise redistribution were independent of the device speed, the RIN spectrum would also be flat but this time at the level of the extreme point of the dip and bump, respectively. Since these speed limitations are due to fundamental carrier dynamics, we expect that they also apply to other regenerators based on self-modulation in SOAs or EAs. This means that the response time of the devices needs to be short in order to get noise redistribution for a large bandwidth. For devices based 


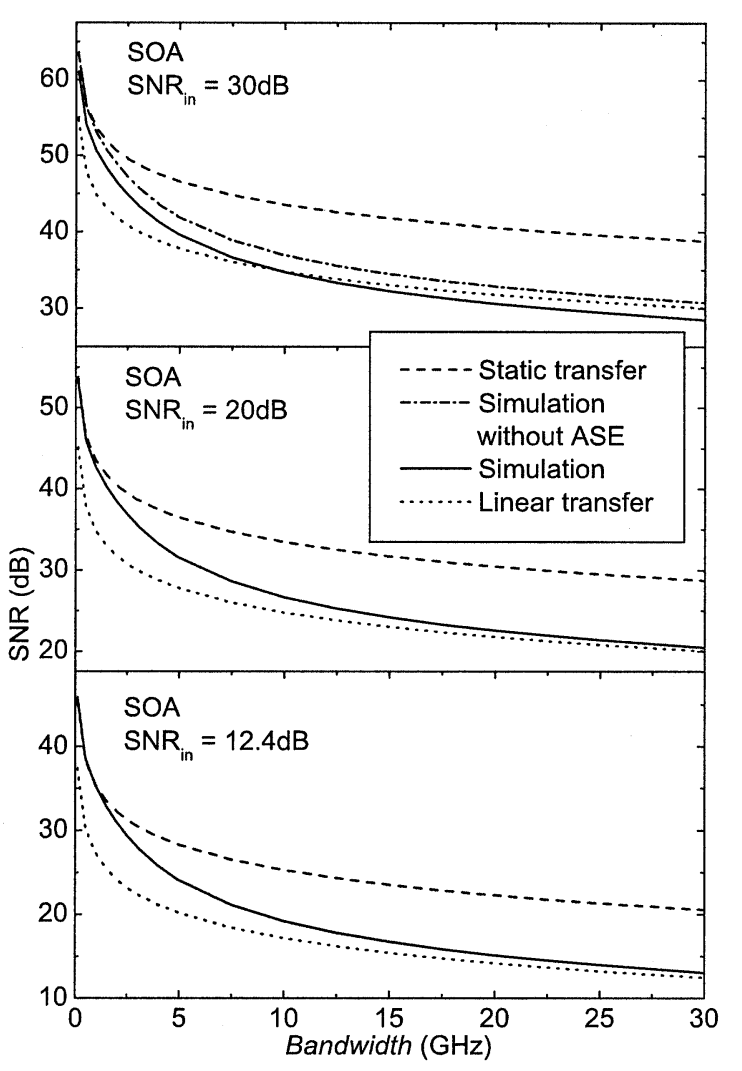

Fig. 4. SNR as a function of detector bandwidth after the SOA. The dynamical models (dashed line) show noise suppression at low bandwidth, approaching the static case (dotted line). At high bandwidth, the SNR approaches the linear case (solid line).

on wavelength conversion, however, other considerations have to be made [28].

Another obvious result is the good agreement between the large-signal simulations and the small-signal analysis. This is explained by the fact that the noise added by the SOA is small compared to the signal power itself, i.e., in the small-signal regime. The noise of the signal at the input to the SOA, on the other hand, is in one case (SNR $=12.4 \mathrm{~dB}$ ) clearly not small. However, the SNR is defined for a $30-\mathrm{GHz}$ bandwidth, which is higher than the modulation bandwidth of the SOA. At these frequencies, the power transfer function is linear and only determined by the gain of the device, shown as the dashed lines in Fig. 1. The mean gain is of course the same in the two models. The noise variance decreases for narrower bandwidth, and within the modulation bandwidth of the SOA the noise of the input signal is still within the small-signal regime.

In order to more clearly see the relation to the limiting cases of linear and static transfer functions we integrate the RIN spectrum to get the SNR. The resulting SNR as a function of bandwidth is shown in Fig. 4 for the SOA and in Fig. 5 for the EA. Different representations of the SOA or EA are considered. The case denoted "linear transfer" is just the linear transformation of the input with the gain at the operation point, while the "static transfer" takes into account the full transfer function. The "simulation" cases are based on the presented dynamical model. For the SOA, results are shown with and without noise added in the SOA for the case of low input

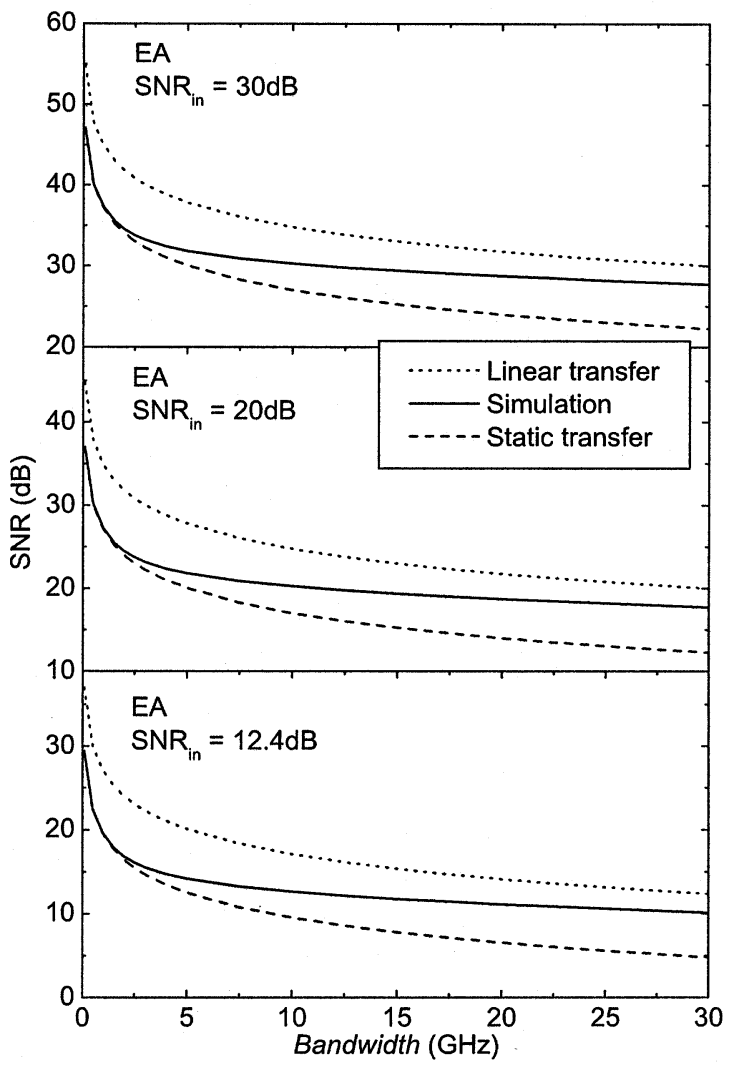

Fig. 5. SNR as a function of detector bandwidth after the EA. The dynamical models (dashed line) show noise enhancement at low bandwidth, approaching the static case (dotted line). At high bandwidth, the SNR approaches the linear case (solid line).

noise $\left(\mathrm{SNR}_{\text {in }}=30 \mathrm{~dB}\right)$. For a lower input SNR, the ASE is negligible and the SNR with and without ASE coincides. Since the simulations and the small-signal calculations give very similar results, as seen previously in the RIN spectrum, only the simulated results have been plotted for the sake of clarity. As the figures show, the nonlinearity of the devices strongly influence their noise properties. In the SOA, both the added ASE and the noise at the input is suppressed at low bandwidths. In the EA, the absorption bleaching acts the opposite way and the SNR is decreased compared to the linear case. The static transfer function only agrees with the simulated and small-signal results at small detection bandwidths, as expected. At higher bandwidth, the SNR approaches that of the linear case instead. This implies that the static transfer function is only useful for cases where the speed of the device is high or for small bandwidths. In the present case, a linear approximation actually gives more accurate results for the SNR, even for moderate bandwidths. However, this limitation is not too severe considering that for a modulated signal the speed of the device has to be high enough to avoid patterning effects, i.e., the device has to have a modulation bandwidth comparable to the bit rate and, hence, the detection bandwidth. The dynamical models, both small-signal and simulation, are on the other hand well suited for calculating the SNR of the output signal at any bandwidth. For the calculation of the BER of a signal, there are, however, other limitations that will be discussed in Section V. 
The conclusions from this section are the understanding of the dynamics of the noise redistribution in the single components and how it approaches the limiting cases of static and linear noise transfer. Furthermore, we demonstrate good agreement between the small-signal analysis and the simulations.

\section{Description of the SOA-EA Device}

We have already, at a few places, referred to the SOA-EA device. In this section, we will, based on the previous section, describe this device in more detail and analyze the dynamics of its noise redistribution.

The device consists of concatenated SOAs and EAs. It is investigated as an inline regenerator and the fiber links and coupling losses are included as a constant loss between the cascaded regenerators, as shown in Fig. 6. In the first pair, the SOA is $800-\mu \mathrm{m}$ long and the EA is $220 \mu \mathrm{m}$, and in the following pairs the lengths are 500 and $295 \mu \mathrm{m}$, respectively. We have investigated devices with one, two, and three SOA-EA pairs in order to further improve the regenerative properties. We have in mind a monolithically integrated device, with forwardand reverse-biased waveguide sections, similar to a modelocked laser design. These devices offer a very simple structure for $2 \mathrm{R}$-regeneration without the complexity of interferometric structures.

By combining the transfer functions of both SOA and EA sections, a nonlinear transfer function is obtained, which should make all-optical regeneration possible. The transfer functions for a single SOA and concatenated SOA-EAs with one, two, and three SOA-EA pairs, including the fiber loss, are shown in Fig. 7. A choice of mark level can be made within the limits given by the gain of the device by changing the amount of fiber loss in the link between the devices. The gain of the device at the mark level, which exactly matches the loss of the fiber, is chosen to be around $10 \mathrm{~dB}$ for all of the examined devices. This choice was made in order to set the decision threshold, i.e., the middle crossing point between the nonlinear transfer function and the linear transfer function, approximately midway between the mark and space level.

These transfer functions regenerate the signal in two different ways. First, as discussed in Section III, a transfer function slope that is less than for the corresponding linear device leads to narrower noise distribution. Second, the absorption bleaching in the EA means that the extinction ratio will be increased. Since the nonlinearity at the " 1 "-level is fairly modest in our example, the extinction ratio improvement is the largest contribution to the regeneration. The increase in extinction ratio from the EA gives a clear advantage compared to the single SOA where the extinction ratio is acctually decreased due to the gain saturation at the "1"-level. Also, a fairly modest nonlinearity can make a substantial difference when the devices are cascaded in a system [29].

Although the extinction ratio increases, it is not obvious that the addition of a saturable absorbing element is advantageous with respect to regeneration. The induced loss has to be balanced by a larger gain, leading to additional noise. However, this is in principle not different from the inevitable insertion loss that has to be outweighed by the degree of reshaping in any regenerator,

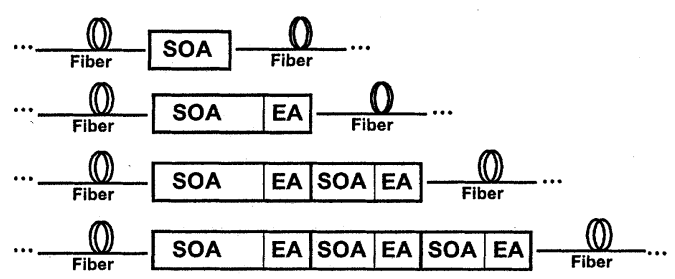

Fig. 6. Simulated module consisting of concatenated SOAs and EAs. The module is considered as an inline regenerator, and the fiber span is considered as a constant loss.

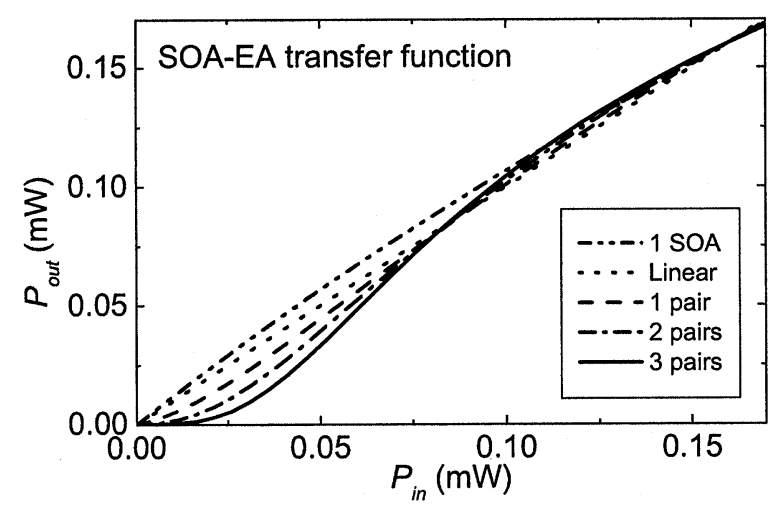

Fig. 7. Static transfer functions of the SOA-EA device, including fiber loss, for one, two, and three SOA-EA pairs. The functions are compared to a linear function.

e.g., interferometric devices. The use of a large gain in the first section of the device helps to limit the noise contribution from the following sections.

Fig. 8 shows the RIN spectrum at the "1"-level of a device with one, two, and three SOA-EA pairs. Fig. 9 shows the SNR for the same situation and compares the SOA-EA devices to the linear transfer and the static transfer function of the three-pair case. As seen from the close-up in the insert of Fig. 8 and also in Fig. 9, the noise is, as expected from the transfer function, reduced for low bandwidth and the reduction is larger for more SOA-EA pairs, although the redistribution is smaller than for the single SOA (see Fig. 2), due to the influence of the EA. The noise enhancement from the EA is also evident and limits the amount of noise redistribution for small bandwidth and leads to a noise enhancement for larger bandwidth. The bandwidth limit, where the noise is enhanced rather than suppressed, is easily seen in Fig. 9 and is about $1.8 \mathrm{GHz}$. As mentioned before, this rather low bandwidth is due to our conservative choice of device parameters and should not be considered to be a performance limit for this type of device in general.

The main advantage of adding more SOA-EA pairs comes from increasing the extinction ratio, while only a modest increase in redistribution at the "1"-level could be seen. The noise enhancement of the EA furthermore limits the bandwidth over which noise suppression can be achieved.

\section{NONLINEAR NOISE REDISTRIBUTION}

The most important noise property when estimating BER is the pdf. Here we will investigate how the SOA-EA re- 


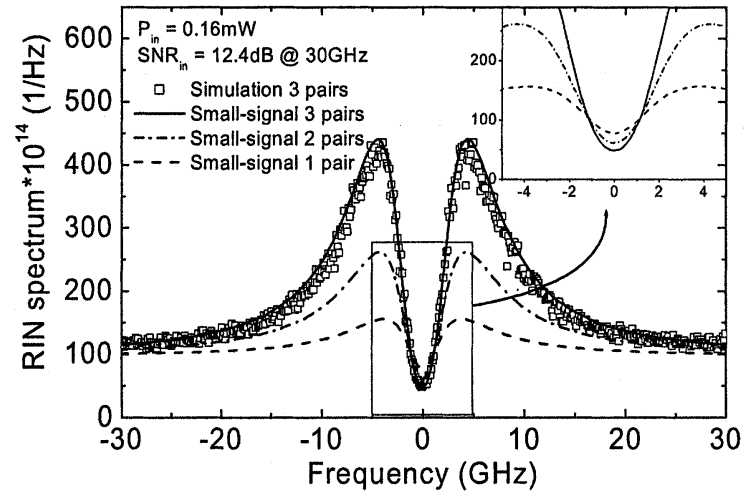

Fig. 8. RIN spectrum of three SOA-EA pairs for an input power of $0.16 \mathrm{~mW}$, corresponding to the mark level. The solid, dash-dotted, and dashed lines represent the small-signal analysis of three, two, and one SOA-EA pairs, respectively. The squares show the simulated data for three pairs. The noise enhancement is due to the EAs and the noise suppression is due to the SOAs. The insert shows a close-up of the improvement in noise redistribution from more pairs, at low frequencies (without the simulation for clarity).

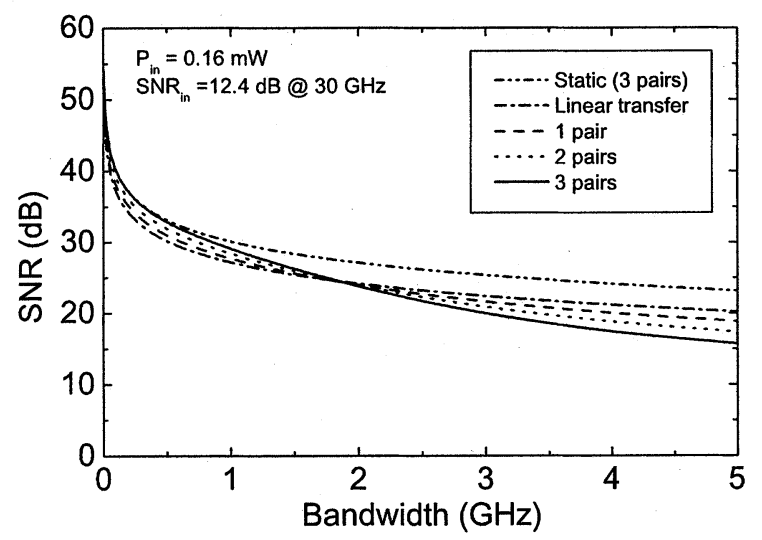

Fig. 9. SNR as a function of detector bandwidth for one, two, and three SOA-EA pairs compared to a linear transfer and the static transfer function of the three-pair device.

distributes the pdf and compare the simulations to standard approximations.

As we have seen in Section III, the small-signal analysis proved to closely match the simulated results for SNR and would thus give a quick way of calculating noise properties of nonlinear devices. However, there is one important feature that the small-signal analysis lacks. Although the SNR gives a good indication of the signal quality, the real estimator of the performance of a system is the BER at the detector. When the SNR is used to estimate the BER, an assumption has to be made regarding the pdf of the signal power since the SNR only provides the variance. The usual assumption is that of a Gaussian or noncentral $\chi^{2}$ distribution [30], [12]. The Gaussian approximation is well known to have several limitations when it comes to properly describing the noise distribution. It does, however, in many cases give good results when used for estimating BER [12], [13] and is widely used. It is therefore included in our comparison for completeness. The redistribution of noise in a nonlinear device, as investigated here, will obviously change the distribution profoundly such that the usual distributions do not apply. For some cases of

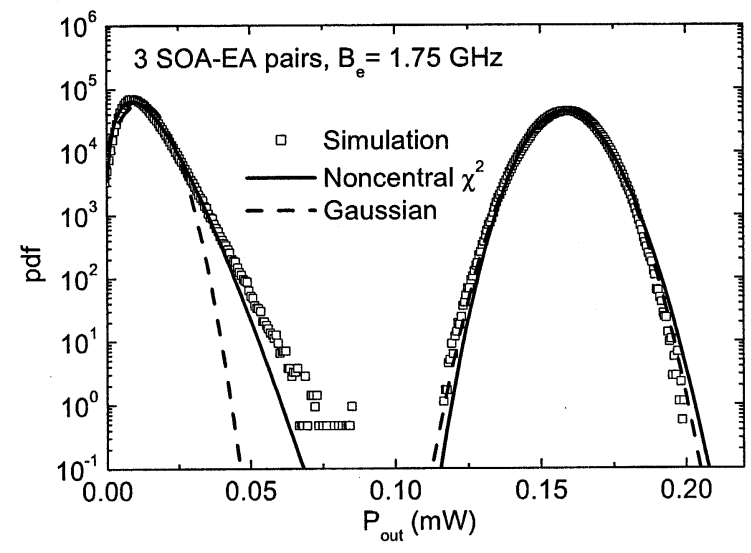

Fig. 10. Noise distribution after three SOA-EA pairs for a bandwidth of $1.75 \mathrm{GHz}$.

nonlinear amplification, this effect is not dominant [9], but for a regenerator it is especially strong in the tails of the distribution, where the nonlinearity is most pronounced, which is also close to the decision threshold where the BER is evaluated. The effect is also larger for a more nonlinear device, i.e., a good regenerator, and it is strongly increased in a cascade of devices. The nonlinear redistribution of the noise can thus result in a pdf and BER that is quite different from the standard approximations employing only the value of the SNR.

If the static transfer function is used to calculate the noise redistribution, the complete pdf can, at least in principle, be calculated, even after a long cascade of devices assuming that the pdf at the input is known. However, this assumption is only justified for low detection bandwidths or high modulation bandwidth of the device.

An example of the nonlinear redistribution of the pdf is shown in Fig. 10, which depicts the noise distribution after a device with three SOA-EA pairs in the form of a histogram. The Gaussian and noncentral $\chi^{2}$ distributions, calculated using the standard deviation given by the SNR, are also plotted for comparison. The mean input power of the cw signal was $0.16 \mathrm{~mW}$ for the mark-level and $0.03 \mathrm{~mW}$ for the space-level, i.e., an extinction ratio of $7.3 \mathrm{~dB}$. The input signal had a noncentral $\chi^{2}$ distribution.

This figure shows that even after a single device with modest nonlinearity the noise distribution differs substantially from the standard approximation at the "0"-level, where the nonlinearity is largest. At the "1"-level, however, the nonlinearity is modest and the approximations fit fairly well with the simulations. Furthermore, the SOA-EA device is capable of improving the extinction ratio from $7.3 \mathrm{~dB}$ at the input to $11.2 \mathrm{~dB}$ at the output.

Due to the excessive computation time required, it is not feasible to simulate the distribution close to the decision threshold where the BER is evaluated. Instead, the nonlinear redistribution can be approximately taken into account, when the BER is evaluated, by tail extrapolation [31]. In this method, the tails of the probability distribution functions are assumed to follow a generalized exponential distribution

$$
f(x ; \nu)=\frac{\nu}{2 \sqrt{2} \sigma \Gamma(1 / \nu)} \exp \left\{-\left|\frac{x-\mu}{\sqrt{2} \sigma}\right|^{\nu}\right\}
$$


where $\Gamma()$ is the gamma function, $\mu$ is the mean value, and the variance of the distribution is given by

$$
\operatorname{Var}=2 \sigma^{2} \frac{\Gamma(3 / \nu)}{\Gamma(1 / \nu)}
$$

A normal (Gaussian) distribution is given by $\nu=2$. By allowing the exponent $\nu$ in the tail to be different from the one in the central part of the distribution, nonlinear behavior can be accounted for. The BER contribution from the " 0 "-level is, as usual, defined as the integral of the distribution above a certain threshold as follows:

$$
\operatorname{BER}(t)=\int_{t+\mu}^{\infty} f(x, \nu) d x
$$

and the contribution from the "1"-level as the usual integral from zero to the threshold. By using the asymptotic expansion for the gamma function, calculating the BER at a few different pseudothresholds $(t)$ in the tail, and fitting the exponent $\nu$ to a plot of the BER versus threshold value, an extrapolation can be made to the actual decision threshold where we want to estimate the BER. This fit can be seen in the inset in Fig. 11 for space and mark levels. The fitting is performed using transformed coordinates so that a linear interpolation using the least-squares technique can be used, as seen in the inset. Fig. 11 shows the BER as a function of the threshold power for space and mark levels. This gives a BER of approximately $10^{-8}$ for the simulated distribution, which should be compared to a BER of $10^{-12}$ for the noncentral $\chi^{2}$ distribution and $10^{-21}$ for the Gaussian approximation. All BERs are calculated with the threshold value at the optimum threshold value for the respective case, as seen in Fig. 11. It should be emphasized that the extrapolated results based on the simulations represents an approximation. However, the large differences in BER show the importance of using the correct noise distribution when dealing with regenerators. Furthermore, this section showed the nonlinear redistribution of the pdf in the SOA-EA device.

\section{CASCADING OF SOA-EA DEVICES}

We have seen that additional SOA-EA pairs in the regenerator give an increase in nonlinearity and better noise redistribution, but also that they add more ASE. In this section, we want to examine how these two effects influence the cascadability of the devices by estimating the BER. We have used two different methods to estimate the regeneration capability of the SOA-EA cascades, the static transfer function and the small-signal analysis. For the static case, the model presented in [14] and [15] was used. The pdfs for marks and spaces are assumed to be known initially and Gaussian noise, representing spontaneous emission for the SOAs, is then added for each link. The amount of noise added, i.e., the standard deviation, is calculated using the small-signal analysis and a choice of bandwidth. The bandwidth limitations will be discussed later. Probability transfer matrices represent the noise redistribution of the nonlinear transfer functions, and concatenation is done by matrix multiplication, which is an effective and fast way to estimate the BER from the pdfs after a cascade of regenerators.

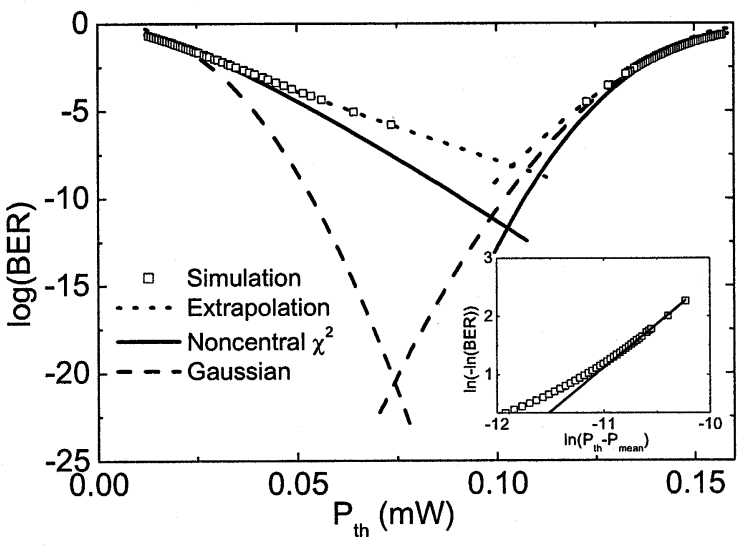

Fig. 11. Tail extrapolation of the simulated noise distributions at mark and space levels. The squares are the simulated data. The lines are the extrapolation for the simulation (dotted line), the Gaussian approximation (dashed line), and the noncentral $\chi^{2}$ distribution (solid line). The inset show the extrapolation technique with the space level of the simulation as an example.

In the small-signal case, the cascadability is investigated by using the $Q$-factor, defined as

$$
Q=\frac{P_{1}-P_{0}}{\sigma_{1}+\sigma_{0}}
$$

where $P_{1}, \sigma_{1}, P_{0}$, and $\sigma_{0}$ are the mean power and standard deviation for the mark and space level, respectively. The small-signal analysis gives us a possibility to examine how the bandwidth dependence of the regeneration influences the cascadability of the devices.

The choices of bandwidth for these calculations require some further comments. In reality, the use of optical filters between the cascaded regenerators are needed in order to limit the growth of ASE power that would otherwise saturate the regenerators. The ASE power is also kept low by the absorption in the EAs. Saturation from ASE is not taken into account in the two models used in this paper. Furthermore, since the static case only involves transfer of pdfs, it is not possible to include the effect of optical bandpass filters with a larger bandwidth than the detection bandwidth. Hence, the important limiting bandwidth is the final detection bandwidth. The standard deviation of the noise added in each regenerator is thus calculated using a detection bandwidth of $2 \mathrm{GHz}$ in the static case. For the small-signal case, no bandwidth limitation is assumed except for the final detection bandwidth, which is chosen to be 1 and $2 \mathrm{GHz}$ in order to show the bandwidth dependence. The choice of such small bandwidth is made in order to be inside the modulation bandwidth of our devices, which is limited by the conservative choice of device parameters, as discussed before.

The result for an input signal with an SNR for the mark level of $25.5 \mathrm{~dB}$ and the same noise variance at the space level is shown in Figs. 12 and 13 for the static and small-signal cases, respectively. The BER (static case) and $Q$-factor (small-signal case) are plotted versus the number of cascaded regenerators for one, two, and three SOA-EA pairs as well as for a single SOA with the same gain as the SOA-EA combinations. In the static case, the obvious result is the improvement in BER for the regenerators compared to the single SOA and linear transfer function due to the increase in extinction ratio by the EAs. For a 


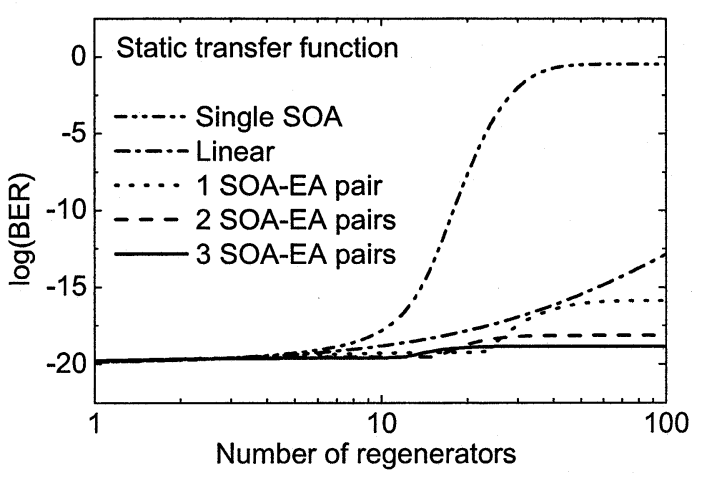

Fig. 12. BER as a function of cascaded fiber links, including SOA-EA regenerators. The BER has been calculated using the static transfer function and ASE noise for one, two, and three SOA-EA pairs. The SNR for the mark level at the input was $25.5 \mathrm{~dB}$ and the noise variance at the space level was the same.

few regenerators, the noise of the input signal dominates, but as more ASE accumulates from several regenerators the BER increases due to the added noise. The BER is evaluated at the optimum threshold value after each link and, due to the larger noise redistribution at the space level compared to the mark level, the threshold value decreases during the first few links as the input noise is redistributed. The rapid increase in the BER that occurs at about 10-20 regenerators comes about when the optimum threshold value cannot decrease further due to the added noise. If instead a fixed threshold is used, this large noise suppression at the space level can not be properly utilized and the BER increases faster due to noise added at the mark level (not shown). A detection with fixed threshold should therefore be combined with a symmetric regenerator where the noise at the space and mark level is redistributed more equally. For a large number of regenerators, the BER only increases slowly due to ASE added in each regenerator, similar to the linear BER degradation with the number of regenerators that is obtained for an ideal step function [14]. It is also seen that the sharper transfer functions do indeed improve the performance of the system for many regenerators even though the additional SOAs gives additional noise.

The small-signal analysis with $B_{e}=2 \mathrm{GHz}$ [(b) in Fig. 13] shows a qualitative behavior somewhat different from the static case. For a few regenerators, the redistribution of the noise at the "0"-level and the increase in extinction ratio keep the $Q$-factor high and more SOA-EA pairs give a better result. However, for many regenerators, the noise at the " 1 "-level decreases the $Q$-factor and the single SOA-EA device gives the best result, as opposed to the static case. For a smaller bandwidth $\left(B_{e}=\right.$ $1 \mathrm{GHz}$, (a) in Fig. 13), the stronger redistribution and smaller ASE make the $Q$-factor increase even for many regenerators. It is also seen that the sharper nonlinear transfer function gives a higher $Q$ for more SOA-EA pairs for the whole cascade at small bandwidth, although the additional SOAs add more noise. Just as in the static case, the lack of extinction ratio improvement in the single SOA gives a worse performance. The reason for the large difference between a detection bandwidth of 1 and $2 \mathrm{GHz}$ can be explained by Fig. 9 where it is easy to see the upper bandwidth limit for noise suppression at the "1"-level.

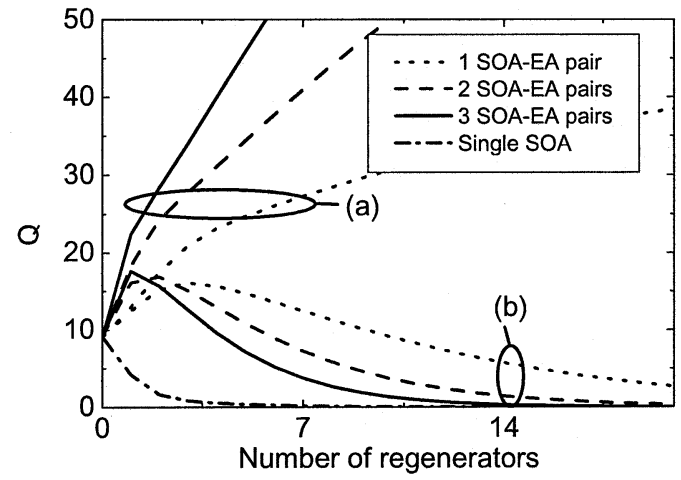

Fig. 13. BER as a function of cascaded fiber links, including SOA-EA regenerators, calculated from the $Q$-factor from the small-signal analysis for a detection bandwidth of (a) $1 \mathrm{GHz}$ and (b) $2 \mathrm{GHz}$.

It should be noted that an increase in $Q$-value does not imply an improvement in BER, only an increase in extinction ration and/or decrease of the noise variances at the " 1 " and/or " 0 "level. To properly calculate the BER, the complete nonlinear transfer of the pdf has to be taken into account, like in the static calculations above.

The conclusion from this is that a sharper nonlinear transfer function can improve the cascadability of the regenerator at small bandwidths, although the means to achieve this, i.e., additional SOA-EA pairs, also adds more noise. A general analysis of this interplay is presented in terms of a general and simplified model in [17] and [29]. Further, we have seen a strong bandwidth dependence of the regenerative properties, hence demonstrating the limitations of the static nonlinear transfer function description, which is only valid for very low data rates. Due to the possibility of having a simple scheme to investigate the cascadability of all-optical regenerators and the possibility of evaluating nonlinear transformation of the pdfs, it is nevertheless very attractive for BER estimations. However, measured and calculated static nonlinear transfer functions should be used with care when analysing all-optical regenerators incorporating SOAs, EAs or, in general, devices with limited modulation bandwidth.

\section{CONCLUSION}

We have theoretically analyzed the noise properties of a device consisting of concatenated SOA and EA sections. This device is predicted to offer significantly improved noise properties compared to a stand-alone SOA and even to have regenerative characteristics. In order to show this, we have examined the detailed dynamics of the noise redistribution in different SOA-EA combinations and compared to the limiting, and often considered, cases of linear and static transfer functions. An example demonstrated the importance of being able to describe the complete nonlinear redistribution of the pdf, when dealing with regenerators, and the shortcomings of the standard approximations in doing so.

\section{REFERENCES}

[1] R. Medina, "Photons vs. electrons [all optical network]," IEEE Potentials, vol. 21, no. 2, pp. 9-11, 2002. 
[2] D. Wolfson, T. Fjelde, A. Kloch, C. Janz, A. Coquelin, I. Guillemot, F. Gaborit, F. Poingt, and M. Renaud, "Experimental investigation at 10 $\mathrm{Gb} / \mathrm{s}$ of the noise suppression capabilities in a pass-through configuration in SOA-based interferometric structures," IEEE Photon. Technol. Lett., vol. 12, pp. 837-839, July 2000.

[3] D. Wolfson, A. Kloch, T. Fjelde, C. Janz, B. Dagens, and M. Renaud, "40-Gb/s all-optical wavelength conversion, regeneration, and demultiplexing in an SOA-based all-active Mach-Zehnder interferometer," IEEE Photon. Technol. Lett., vol. 12, pp. 332-334, Mar. 2000.

[4] M. Zhao, J. De Merlier, G. Morthier, and R. Baets, "Experimental demonstration at $10 \mathrm{Gbps}$ of $2 \mathrm{R}$ optical regeneration in a fiber-based MZI with LOAs," in Proc. ECOC, vol. 3, 2002, p. 7.3.6.

[5] J. De Merlier, G. Morthier, S. Verstuyft, T. Van Caenegem, I. Moerman, P. Van Daele, and R. Baets, "Experimental demonstration of all-optical regeneration using an MMI-SOA," IEEE Photon. Technol. Lett., vol. 14, pp. 660-662, May 2002.

[6] C. Knöll, M. Gölles, Z. Bakonyi, G. Onishchukov, and F. Lederer, "Optimization of signal transmission by an in-line semiconductor optical amplifier-saturable absorber module," Opt. Commun., vol. 187, no. 1, pp. 141-153, 2001.

[7] T. Watanabe, N. Sakaida, H. Yasaka, F. Kano, and M. Koga, "Transmission performance of chirp-controlled signal by using semiconductor optical amplifier," J. Lightwave Technol., vol. 18, pp. 1069-1077, Aug. 2000.

[8] K. Inoue, "Technique to compensate waveform distortion in a gain-saturated semiconductor optical amplifier using a semiconductor saturable absorber," Electron. Lett., vol. 34, no. 4, pp. 376-378, 1998.

[9] M. Shtaif and G. Eisenstein, "Experimental study of the statistical properties of nonlinearly amplified signals in semiconductor optical amplifiers," IEEE Photon. Technol. Lett., vol. 9, pp. 904-906, July 1997.

[10] N. Olsson, "Lightwave systems with optical amplifiers," J. Lightwave Technol., vol. 7, pp. 1071-1082, July 1989

[11] M. Shtaif, B. Tromborg, and G. Eisenstein, "Noise spectra of semiconductor optical amplifiers: Relation between semiclassical and quantum descriptions," IEEE J. Quantum Electron., vol. 34, pp. 869-878, May 1998.

[12] G. H. Einarsson, Principles of Lightwave Communication, 1st ed. New York: Wiley, 1996.

[13] P. Humblet and M. Azizoglu, "On the bit error rate of lightwave systems with optical amplifiers," J. Lightwave Technol., vol. 9, pp. 1576-1586, Nov. 1991.

[14] P. Öhlen and E. Berglind, "Noise accumulation and BER estimates in concatenated nonlinear optoelectronic repeaters," IEEE Photon Technol. Lett., vol. 9, pp. 1011-1013, July 1997.

[15] S. Bischoff, B. Lading, and J. Mørk, "BER estimation for all-optical regenerators influenced by pattern effects," IEEE Photon. Technol. Lett., vol. 14, pp. 33-35, Jan. 2002.

[16] K. Obermann and K. Petermann, "Estimation of BER performance and cascadability of wavelength converters based on cross-gain modulation in semiconductor optical amplifiers," Proc. Inst. Elect. Eng., pt. J, vol. 147, no. 2, pp. 133-137, 2000.

[17] J. Mørk, F. Öhman, and S. Bischoff, "Analytical BER-expression for cascaded regenerators and analysis of nonlinearity versus noise," in Proc. LEOS, vol. 2, 2002, pp. 895-896.

[18] G. Agrawal and N. Olsson, "Self-phase modulation and spectral broadening of optical pulses in semiconductor laser amplifiers," IEEE J. Quantum Electron., vol. 25, pp. 2297-2306, Nov. 1989.

[19] D. Cassioli, S. Scotti, and A. Mecozzi, "A time-domain computer simulator of the nonlinear response of semiconductor optical amplifiers," IEEE J. Quantum Electron., vol. 36, pp. 1072-1080, Sept. 2000.

[20] S. Højfeldt, S. Bischoff, and J. Mørk, "All-optical wavelength conversion and signal regeneration using an electroabsorption modulator," $J$. Lightwave Technol., vol. 18, pp. 1121-7, Aug. 2000.

[21] J. Mørk and A. Mecozzi, "Theory of the ultrafast optical response of active semiconductor waveguides," J. Opt. Soc. Amer. B, vol. 13, no. 8, pp. 2437-2452, 1996.

[22] Z. Bakonyi, G. Onishchukov, C. Knöll, M. Gölles, F. Lederer, and R. Ludwig, "In-line saturable absorber in transmission systems with cascaded semiconductor optical amplifiers," IEEE Photon. Technol. Lett., vol. 12 , pp. 570-572, May 2000.

[23] A. D. Ellis, A. E. Kelly, D. Nesset, D. Pitcher, and D. G. Moodie, "Erro free $100 \mathrm{Gbit} / \mathrm{s}$ wavelength conversion using grating assisted cross-gain modulation in $2 \mathrm{~mm}$ long semiconductor amplifier," Electron. Lett., vol. 34, no. 20, pp. 1958-1959, 1998.

[24] H. Tanaka, M. Hayashi, T. Otani, K. Ohara, and M. Suz, "60 Gbit/s WDM-OTDM transmultiplexing using an electro-absorption modulator," in Proc. Optical Fiber Communication Conf. and Exhibit, vol. 1, 2001, pp. ME4/1-ME4/3.
[25] K. Sato and H. Toba, "Reduction of mode partition noise by using semiconductor optical amplifiers," IEEE J. Quantum Electron., vol. 37, pp. 328-333, Feb. 2001.

[26] M. Zhao, G. Morthier, and R. Baets, "Analysis and optimization of intensity noise reduction in spectrum-sliced WDM systems using a saturated semiconductor optical amplifier," IEEE Photon. Technol. Lett., vol. 14, pp. 390-392, Mar. 2002.

[27] H. Yokoyama, Y. Hashimoto, and H. Kurita, "Noise reduction in optical pulses and bit-error-rate improvement with a semiconductor-waveguide saturable absorber," in Tech. Dig. CLEO'98, pp. 502-503.

[28] K. Obermann, S. Kindt, D. Breuer, K. Petermann, C. Schmidt, S. Diez, and H. Weber, "Noise characteristics of semiconductor-optical amplifiers used for wavelength conversion via cross-gain and cross-phase modulation," IEEE Photon. Technol. Lett., vol. 9, pp. 312-314, Mar. 1997.

[29] J. Mørk, F. Öhman, and S. Bischoff, "Analytical expression for the bit error rate of cascaded all-optical regenerators," IEEE Photon. Technol. Lett., vol. 15, pp. 1479-1481, Oct. 2003.

[30] G. P. Agrawal, Fiber-Optic Communication Systems, 2nd ed. New York: Wiley-Interscience, 1997.

[31] M. C. Jeruchim, P. Balaban, and K. S. Shanmugan, Simulation of Communication Systems, Modeling, Methodology and Techniques, 2nd ed. New York: Kluwer/Plenum, 2000.

Filip Öhman received the M.Sc. degree in engineering from the Royal Institute of Technology (KTH), Stockholm, Sweden, in 2001. He is currently working toward the Ph.D. degree at the Technical University of Denmark, Lyngby.

His research interests are optical signal processing and noise in nonlinear semiconductor devices.

Svend Bischoff received the M.Sc. degree in engineering and the Ph.D. degree from the Technical University of Denmark, Lyngby, in 1993 and 1997, respectively.

From 1998 to 2002, he was with the Research Center for Communications, Optics and Materials (COM), Technical University of Denmark, which he left in 2002 to join Alight Technologies, Farum, Denmark. His research interests are optical signal processing and ultrafast dynamics in semiconductor devices, electroabsorption modulators, and quantum-dot devices.

Bjarne Tromborg was born in 1940 in Denmark. He received the M.Sc. degree in physics and mathematics from the Niels Bohr Institute, Copenhagen, Denmark, in 1968.

He was a university researcher in high-energy particle physics, and for one year a high school teacher, until he joined the research laboratory of the Danish Teleadministrations in 1979. He was Head of Optical Communications Department, Tele Danmark Research, from 1987 to 1995, Adjunct Professor at the Niels Bohr Institute from 1991 to 2001, Project Manager in Tele Danmark R\&D from 1996 to 1998, and on a leave of absence at the Technion, Haifa, Israel, in 1997. He is now a Professor in modeling of components and systems for optical communications at the Research Center for Communications, Optics and Materials (COM), Technical University of Denmark, Lyngby. He has coauthored a research monograph and more than 90 journal and conference publications, mostly on physics and technology of optoelectronic devices.

Prof. Tromborg was the recipient of the Electro-prize from the Danish Society of Engineers in 1981. He is an Associate Editor of the IEEE JOURNAL OF QUANTUM ELECTRONICS.

Jesper Mørk received the M.Sc. and Ph.D. degrees from the Technical University of Denmark, Lyngby, in 1986 and 1988, respectively.

Since 2002, he has been a Professor in semiconductor devices for optical communication systems and is responsible for modeling and theory in the optoelectronics competence area at the Research Center for Communications, Optics and Materials (COM), Technical University of Denmark. His current research interests are in the area of device physics, in particular ultrafast devices for optical signal processing, quantum dot devices, and noise in nonlinear devices. 